\title{
AN ANALYSIS OF A FRACTAL KINETICS CURVE OF SAVAGEAU
}

\author{
JOHN MALONEY ${ }^{1}$ and JACK HEIDEL ${ }^{1}$
}

(Received 26 June, 2000; revised 21 January, 2001)

\begin{abstract}
The fractal kinetics curve derived by Savageau is analysed to show that its parameters are not uniquely determined given four appropriately situated data points. Comparison is made with an alternate fractal Michaelis-Menten equation derived by Lopez-Quintela and Casado.
\end{abstract}

\section{Introduction}

The Michaelis-Menten equation

$$
V_{p}=\frac{V_{m} S}{S+K_{m}}
$$

has long been the standard framework for biochemical kinetics [4], describing the reaction of a substrate $S$ on a free enzyme $E$ to form a product $P$. Here $V_{p}=d P / d t$ and $V_{m}$ and $K_{m}$ are empirically determined parameters. It is convenient to rewrite this equation in the form

$$
\left(\frac{S}{K_{m}}\right)=\left(\frac{V_{p}}{V_{m}}\right)\left(1-\frac{V_{p}}{V_{m}}\right)^{-1}
$$

If the reaction is allosteric (cooperative) so that $n$ molecules of $S$ bind to $E$, the kinetics are described by the Hill equation [5]

$$
V_{p}=\frac{V_{m} S^{n}}{K_{m}+S^{n}}
$$

\footnotetext{
${ }^{1}$ Department of Mathematics, University of Nebraska at Omaha, Omaha, Nebraska 68182, USA; e-mail: maloney@unomaha.edu,jheidel@unomaha.edu.

(C) Australian Mathematical Society 2003, Serial-fee code 1446-1811/03
} 
which can be written

$$
\left(\frac{S^{n}}{K_{m}}\right)=\left(\frac{V_{p}}{V_{m}}\right)\left(1-\frac{V_{p}}{V_{m}}\right)^{-1} .
$$

Recently, Savageau [6] has shown that both the Michaelis-Menten and Hill equations are special cases of a more general fractal power law mechanism which produces the kinetic equation

$$
\left(\frac{S}{K_{f}}\right)^{g_{s}}=\left(\frac{V_{p}}{V_{m}}\right)\left(1-\frac{V_{p}}{V_{m}}\right)^{-g_{e}} .
$$

Clearly, (1.3) includes (1.1) and (1.2) as special cases where $g_{s}$ and $g_{e}$ can be interpreted as generalised cooperativity exponents. In fact, $g_{e}=1$ reduces (1.3) to the Hill equation (1.2).

Note that (1.1) has two parameters $V_{m}$ and $K_{m},(1.2)$ has three parameters $V_{m}$, $K_{m}$ and $n$ while (1.3) has four parameters $V_{m}, K_{f}, g_{s}$ and $g_{e}$. Recently, the authors [1] have made a data-fitting analysis of (1.1) and (1.2). For the Michaelis-Menten equation (1.1) let $\left(V_{p_{1}}, S_{1}\right)$ and $\left(V_{p_{2}}, S_{2}\right)$ be two points on the hyperbolic (concave down) response curve described by (1.1). Then these two pieces of data uniquely determine the parameters $V_{m}$ and $K_{m}$.

For the Hill equation (1.2) three data points $\left(V_{p_{1}}, S_{1}\right),\left(V_{p_{2}}, S_{2}\right)$ and $\left(V_{p_{3}}, S_{3}\right)$ either determine the three parameters $V_{m}, K_{m}$ and $n$ uniquely or there may be no solution for the three parameters. It depends on a precisely determined but complicated relationship between the three data points [1].

The purpose of this paper is to carry out a similar analysis for the more general equation (1.3) with four parameters. It turns out that there will always be two, infinitely many, or no solutions for the four parameters, given four data points. That is, it is impossible to have a single unique solution for the four parameters regardless of the specified data.

Before beginning the analysis of (1.3), another fractal generalisation of the Michaelis-Menten equation should be mentioned. By assuming that the reaction rate constants $K_{i}$ are scale dependent, $K_{i}=A_{i} S^{1-D}$ where $D \leq 1$ is the fractal dimension of the scaling variable $S$, Lopez-Quintela and Casado [3] were led to the equation

$$
V_{p}=\frac{V_{m}^{\mathrm{eff}} S^{2-D}}{K_{m}^{\mathrm{eff}}+S},
$$

which is distinct from (1.2) and (1.3) above. The data-fitting problem for (1.4) has been analysed in [2]. It turns out that for this three-parameter equation there can also be either one, two, or no solutions depending in a complicated way on the alignment of the data points. However, a unique solution exists only if the three data points lie 
on a single special curve separating the region of no solutions from the regions of two solutions ([2, Curve (11) in Figure 7]).

Thus the two papers, [3] and [6], propose different models for fractal reaction kinetics. In one model there exists a single data curve producing a unique solution for the parameters, which is not robust. In the other model, there is never a unique solution for the system parameters. Thus data analysis of the two models uncovers shortcomings in each one and raises the question as to whether either is an adequate model of reality.

This is a theoretical analysis, assuming only a minimal amount of data is available to determine the parameters of the model. Such an analysis is a first step in evaluating the practical utility of a model. It is clearly important to know whether or not the model parameters are uniquely determined by data even if only in a theoretical setting. Then the problems of parameter estimation and sensitivity analysis can be carried out later if the model is applied to specific sets of data.

\section{Results}

We now turn to the analysis of (1.3). For ease of notation we use $V_{i}$ instead of $V_{p_{i}}$.

THEOREM 1. Given four data points $\left(V_{i}, S_{i}\right), i=1,2,3,4$, where $0<V_{1}<\cdots<$ $V_{4}$ and $0<S_{1}<\cdots<S_{4}$, then there either exists one, two, infinitely many, or no solutions for $V_{m}, K_{f}, g_{s}$ and $g_{e}$, where $V_{m}$ and $K_{f}$ are assumed to be positive.

Proof. Substitute $\left(V_{i}, S_{i}\right), i=1,2,3,4$, into (1.3) for $\left(V_{i}, S_{i}\right)$ and take logarithms of both sides to obtain

$$
g_{s}\left(\ln S_{i}-\ln K_{f}\right)=\ln V_{i}-\left(1-g_{e}\right) \ln V_{m}-g_{e} \ln \left(V_{m}-V_{i}\right) .
$$

Solving for $\ln K_{f}$ gives

$$
\ln K_{f}=\frac{1}{g_{s}} \ln \left(\frac{V_{m}}{V_{i}}\right)+\frac{g_{e}}{g_{s}} \ln \left(\frac{V_{m}-V_{i}}{V_{m}}\right)+\ln S_{i} .
$$

Subtracting the first two of these four equations (that is, for $i=1$ and 2) eliminates the $K_{f}$ term and gives

$$
\frac{1}{g_{s}} \ln \left(\frac{V_{2}}{V_{1}}\right)+\frac{g_{e}}{g_{s}} \ln \left(\frac{V_{m}-V_{1}}{V_{m}-V_{2}}\right)+\ln \left(\frac{S_{1}}{S_{2}}\right)=0 .
$$

Similarly using $i=1$ and 3 gives

$$
\frac{1}{g_{s}} \ln \left(\frac{V_{3}}{V_{1}}\right)+\frac{g_{e}}{g_{s}} \ln \left(\frac{V_{m}-V_{1}}{V_{m}-V_{3}}\right)+\ln \left(\frac{S_{1}}{S_{3}}\right)=0 .
$$


These two equations can be solved for $g_{e}$ and $g_{s}$ to give

and

$$
g_{e}=\frac{\ln \left(\frac{s_{1}}{s_{2}}\right) \ln \left(\frac{V_{2}}{V_{3}}\right)-\ln \left(\frac{V_{2}}{V_{1}}\right) \ln \left(\frac{s_{3}}{s_{2}}\right)}{\ln \left(\frac{s_{1}}{s_{2}}\right) \ln \left(\frac{V_{m}-V_{2}}{V_{m}-V_{3}}\right)+\ln \left(\frac{V_{m}-V_{1}}{V_{m}-V_{2}}\right) \ln \left(\frac{s_{3}}{s_{2}}\right)}
$$

$$
g_{s}=\frac{\ln \left(\frac{V_{m}-V_{1}}{V_{m}-V_{2}}\right) \ln \left(\frac{V_{3}}{V_{1}}\right)-\ln \left(\frac{V_{m}-V_{1}}{V_{m}-V_{3}}\right) \ln \left(\frac{V_{2}}{V_{1}}\right)}{\ln \left(\frac{s_{1}}{S_{2}}\right) \ln \left(\frac{V_{m}-V_{2}}{V_{m}-V_{3}}\right)+\ln \left(\frac{V_{m}-V_{1}}{V_{m}-V_{2}}\right) \ln \left(\frac{S_{3}}{S_{2}}\right)} .
$$

Thus $K_{f}, g_{e}$ and $g_{s}$ are all expressed in terms of $V_{m}$ and the data points $\left(V_{i}, S_{i}\right)$. Since we haven't yet used ( $i=1$ and 4)

$$
\frac{1}{g_{s}} \ln \left(\frac{V_{4}}{V_{1}}\right)+\frac{g_{e}}{g_{s}} \ln \left(\frac{V_{m}-V_{1}}{V_{m}-V_{4}}\right)+\ln \left(\frac{S_{1}}{S_{4}}\right)=0
$$

we can substitute into this the expressions for $g_{e}$ and $g_{s}$ to obtain

$$
\begin{aligned}
& \frac{\ln \left(\frac{S_{1}}{S_{2}}\right) \ln \left(\frac{V_{m}-V_{2}}{V_{m}-V_{3}}\right)+\ln \left(\frac{V_{m}-V_{1}}{V_{m}-V_{2}}\right) \ln \left(\frac{S_{2}}{S_{2}}\right)}{\ln \left(\frac{V_{m}-V_{1}}{V_{m}-V_{2}}\right) \ln \left(\frac{V_{3}}{V_{1}}\right)-\ln \left(\frac{V_{m}-V_{1}}{V_{m}-V_{3}}\right) \ln \left(\frac{V_{2}}{V_{1}}\right)} \ln \left(\frac{V_{4}}{V_{1}}\right) \\
& \quad+\frac{\ln \left(\frac{S_{1}}{S_{2}}\right) \ln \left(\frac{V_{2}}{V_{3}}\right)-\ln \left(\frac{V_{2}}{V_{1}}\right) \ln \left(\frac{S_{3}}{S_{2}}\right)}{\ln \left(\frac{V_{m}-V_{1}}{V_{m}-V_{2}}\right) \ln \left(\frac{V_{3}}{V_{1}}\right)-\ln \left(\frac{V_{m}-V_{1}}{V_{m}-V_{3}}\right) \ln \left(\frac{V_{2}}{V_{1}}\right)} \ln \left(\frac{V_{m}-V_{1}}{V_{m}-V_{4}}\right)+\ln \left(\frac{S_{1}}{S_{4}}\right)=0 .
\end{aligned}
$$

This equation can be rewritten as

$$
\alpha_{1} \ln \left(V_{m}-V_{1}\right)+\alpha_{2} \ln \left(V_{m}-V_{2}\right)+\alpha_{3} \ln \left(V_{m}-V_{3}\right)+\alpha_{4} \ln \left(V_{m}-V_{4}\right)=0
$$

or

$$
\left(V_{m}-V_{1}\right)^{\alpha_{1}}\left(V_{m}-V_{2}\right)^{\alpha_{2}}\left(V_{m}-V_{3}\right)^{\alpha_{3}}\left(V_{m}-V_{4}\right)^{\alpha_{4}}=1,
$$

where

$$
\begin{aligned}
& \alpha_{1}=\ln \left(\frac{S_{3}}{S_{4}}\right) \ln \left(V_{2}\right)+\ln \left(\frac{S_{4}}{S_{2}}\right) \ln \left(V_{3}\right)+\ln \left(\frac{S_{2}}{S_{3}}\right) \ln \left(V_{4}\right), \\
& \alpha_{2}=\ln \left(\frac{S_{4}}{S_{3}}\right) \ln \left(V_{1}\right)+\ln \left(\frac{S_{1}}{S_{4}}\right) \ln \left(V_{3}\right)+\ln \left(\frac{S_{3}}{S_{1}}\right) \ln \left(V_{4}\right), \\
& \alpha_{3}=\ln \left(\frac{S_{2}}{S_{4}}\right) \ln \left(V_{1}\right)+\ln \left(\frac{S_{4}}{S_{1}}\right) \ln \left(V_{2}\right)+\ln \left(\frac{S_{1}}{S_{2}}\right) \ln \left(V_{4}\right), \\
& \alpha_{4}=\ln \left(\frac{S_{3}}{S_{2}}\right) \ln \left(V_{1}\right)+\ln \left(\frac{S_{1}}{S_{3}}\right) \ln \left(V_{2}\right)+\ln \left(\frac{S_{2}}{S_{1}}\right) \ln \left(V_{3}\right)
\end{aligned}
$$




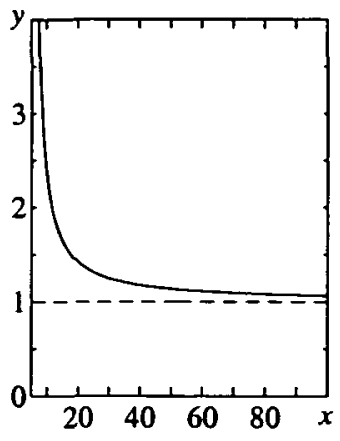

(a)

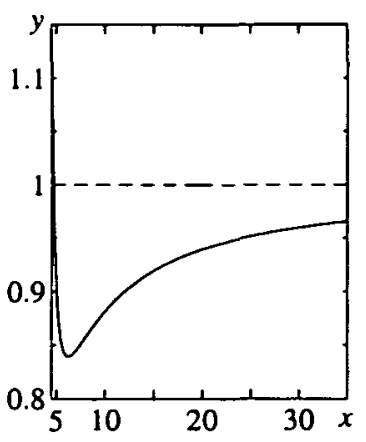

(b)

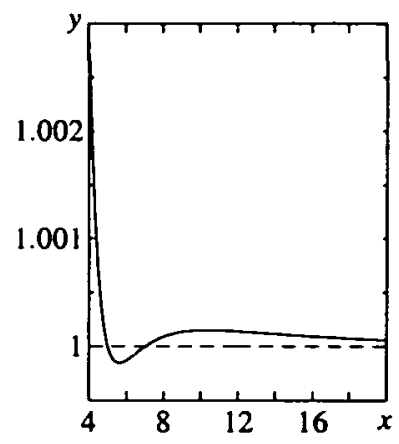

(c)

FIGURE 1. Graph of $h\left(V_{m}\right)$ with $h\left(V_{4}^{+}\right)=\infty$ where the line $y=1$ is intersected (a) zero times, (b) once or (c) twice.

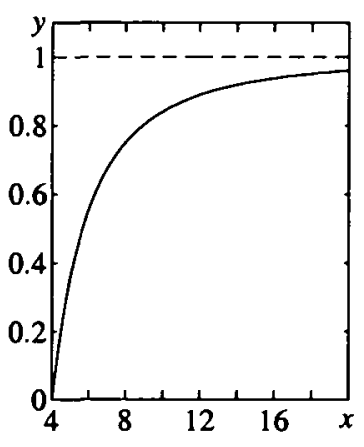

(a)

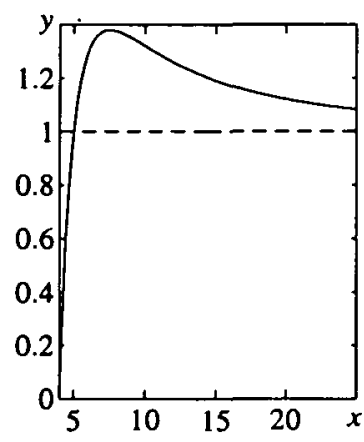

(b)

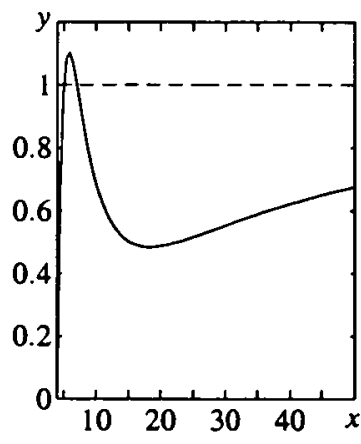

(c)

FIGURE 2. Graph of $h\left(V_{m}\right)$ with $h\left(V_{4}^{+}\right)=0$ where the line $y=1$ is intersected (a) zero times, (b) once or (c) twice.

and $\alpha_{1}+\alpha_{2}+\alpha_{3}+\alpha_{4}=0$.

We note that $V_{m}$ represents the maximum velocity in (1.1)-(1.3). Then with $V_{m}>V_{i}$ for $i=1,2,3,4$ and using the fact that $\alpha_{4}=-\alpha_{1}-\alpha_{2}-\alpha_{3}$, we set

$$
h\left(V_{m}\right) \equiv\left(\frac{V_{m}-V_{1}}{V_{m}-V_{4}}\right)^{\alpha_{1}}\left(\frac{V_{m}-V_{2}}{V_{m}-V_{4}}\right)^{\alpha_{2}}\left(\frac{V_{m}-V_{3}}{V_{m}-V_{4}}\right)^{\alpha_{3}}
$$

and note that (2.1) is equivalent to finding a $V_{m}$ such that $h\left(V_{m}\right)=1$. We also note that $h\left(V_{m}\right) \equiv 1$ if and only if $\alpha_{1}=\alpha_{2}=\alpha_{3}=\alpha_{4}=0$, in which case there are infinitely many solutions.

It is easy to show that

$$
h^{\prime}\left(V_{m}\right)=\frac{h\left(V_{m}\right) Q\left(V_{m}\right)}{\left(V_{m}-V_{4}\right)\left(V_{m}-V_{3}\right)\left(V_{m}-V_{2}\right)\left(V_{m}-V_{1}\right)},
$$


where $Q\left(V_{m}\right)$ is a quadratic in $V_{m}$ whose coefficients are combinations of $V_{i}$ 's and $\alpha_{i}$ 's. Since $h\left(V_{m}\right)>0$ on its domain $\left(V_{4}, \infty\right)$ and assuming $h\left(V_{m}\right) \not \equiv 1, \lim _{V_{m} \rightarrow V_{4}} h\left(V_{m}\right)=$ $h\left(V_{4}^{+}\right)=\infty$ or 0 and $\lim _{V_{m} \rightarrow \infty} h\left(V_{m}\right)=h(\infty)=1$, then $h^{\prime}\left(V_{m}\right)$ changes sign at most twice and the graph of $h\left(V_{m}\right)$ has one of the forms shown in Figure 1 for $h\left(V_{4}^{+}\right)=\infty$ and in Figure 2 for $h\left(V_{4}^{+}\right)=0$.

It is thus clear that $h\left(V_{m}\right)$ intersects the line $y=1$ either once, twice, or no times and the theorem is proved.

To proceed further, we need to know the coefficient of the squared term in $Q\left(V_{m}\right)$. It is (found by MAPLE)

$$
\begin{aligned}
\alpha= & \left(V_{1}-V_{4}\right) \alpha_{1}+\left(V_{2}-V_{4}\right) \alpha_{2}+\left(V_{3}-V_{4}\right) \alpha_{3} \\
= & \left(V_{1}-V_{4}\right)\left(\ln \left(\frac{S_{3}}{S_{4}}\right) \ln \left(V_{2}\right)+\ln \left(\frac{S_{4}}{S_{2}}\right) \ln \left(V_{3}\right)+\ln \left(\frac{S_{2}}{S_{3}}\right) \ln \left(V_{4}\right)\right) \\
& +\left(V_{2}-V_{4}\right)\left(\ln \left(\frac{S_{4}}{S_{3}}\right) \ln \left(V_{1}\right)+\ln \left(\frac{S_{1}}{S_{4}}\right) \ln \left(V_{3}\right)+\ln \left(\frac{S_{3}}{S_{1}}\right) \ln \left(V_{4}\right)\right) \\
& +\left(V_{3}-V_{4}\right)\left(\ln \left(\frac{S_{2}}{S_{4}}\right) \ln \left(V_{1}\right)+\ln \left(\frac{S_{4}}{S_{1}}\right) \ln \left(V_{2}\right)+\ln \left(\frac{S_{1}}{S_{2}}\right) \ln \left(V_{4}\right)\right) .
\end{aligned}
$$

It turns out that the question of the existence of a unique solution of (2.1), that is, a unique $V_{m}$ such that $h\left(V_{m}\right)=1$, can be resolved completely using only $\alpha$, the coefficient of the quadratic term in $Q\left(V_{m}\right)$. It is clear from Figure 1 that when $h\left(V_{4}^{+}\right)=\infty$, there is a unique solution if $\alpha>0$, and either two solutions or no solution if $\alpha<0$. In a similar manner it is clear from Figure 2 that when $h\left(V_{4}^{+}\right)=0$, there is a unique solution if $\alpha<0$ and either two solutions or no solution if $\alpha>0$.

Of course, the expression for $\alpha$ involves $\alpha_{1}, \alpha_{2}$ and $\alpha_{3}$. It turns out that the sign of each of the $\alpha_{i}$ has a simple and useful geometric interpretation.

LEMMA 1. The parameter $\alpha_{1}>0(=0),(<0)$ if and only if $\left(\ln V_{3}, \ln S_{3}\right)$ lies above (on), (below) the line from $\left(\ln V_{2}, \ln S_{2}\right)$ to $\left(\ln V_{4}, \ln S_{4}\right)$.

Proof. Suppose that

$$
\begin{aligned}
0=\alpha_{1} & =\left(\ln S_{3}-\ln S_{4}\right) \ln V_{2}+\left(\ln S_{4}-\ln S_{2}\right) \ln V_{3}+\left(\ln S_{2}-\ln S_{3}\right) \ln V_{4} \\
& =\ln \frac{V_{2}}{V_{4}} \ln S_{3}+\ln \frac{S_{4}}{S_{2}} \ln V_{3}+\ln S_{2} \ln V_{4}-\ln S_{4} \ln V_{2} .
\end{aligned}
$$

Thus

$$
\ln S_{3}-\ln V_{3} \frac{\ln S_{4}-\ln S_{2}}{\ln V_{4}-\ln V_{2}}=\frac{\ln S_{2} \ln V_{4}-\ln S_{4} \ln V_{2}}{\ln V_{4}-\ln V_{2}}
$$


or

$$
\ln S_{3}=\frac{\ln S_{4}-\ln S_{2}}{\ln V_{4}-\ln V_{2}} \ln V_{3}+\ln S_{2}-\frac{\ln S_{4}-\ln S_{2}}{\ln V_{4}-\ln V_{2}} \ln V_{2},
$$

which is the line $y=m x+b$ with $y=\ln S_{3}$ and $x=\ln V_{3}$ between $\left(\ln V_{2}, \ln S_{2}\right)$ and $\left(\ln V_{4}, \ln S_{4}\right)$.

Since we have divided by a positive number, the two inequalities follow directly.

In a completely analogous manner the following geometric interpretation of $\alpha_{2}, \alpha_{3}$ and $\alpha_{4}$ can also be established.

LEMMA 2. The parameter $\alpha_{2}>0(=0),(<0)$ if and only if $\left(\ln V_{3}, \ln S_{3}\right)$ lies above (on), (below) the line from $\left(\ln V_{1}, \ln S_{1}\right)$ to $\left(\ln V_{4}, \ln S_{4}\right)$.

LEMMA 3. The parameter $\alpha_{3}>0(=0),(<0)$ if and only if $\left(\ln V_{2}, \ln S_{2}\right)$ lies above (on), (below) the line from $\left(\ln V_{1}, \ln S_{1}\right)$ to $\left(\ln V_{4}, \ln S_{4}\right)$.

LEMMA 4. The parameter $\alpha_{4}>0(=0),(<0)$ if and only if $\left(\ln V_{2}, \ln S_{2}\right)$ lies above (on), (below) the line from $\left(\ln V_{1}, \ln S_{1}\right)$ to $\left(\ln V_{4}, \ln S_{4}\right)$.

We are now able to establish the main result of this paper.

THEOREM 2. There is never a unique set of parameters for (1.3). Otherwise stated, (2.1), $h\left(V_{m}\right)=1$, never has a unique solution $V_{m}$.

Proof. We adopt the notation $(+,-,+,-)$, for example, to indicate that $\alpha_{1}>0$, $\alpha_{2}<0, \alpha_{3}>0$ and $\alpha_{4}<0$. Similarly a + or - replaced by a zero indicates that the corresponding component is zero.

First suppose that $\alpha_{1}+\alpha_{2}+\alpha_{3}>0$ so that $h\left(V_{4}^{+}\right)=\infty$ and Figure 1 applies. Thus also $\alpha_{4}<0$ since $\alpha_{1}+\alpha_{2}+\alpha_{3}+\alpha_{4}=0$. We now have to consider various cases for the sign possibilities for $\alpha_{1}, \alpha_{2}$ and $\alpha_{3}$ (with $\alpha_{4}<0$ ).

Case 1: $(+,-,-,-)$. Since $\alpha_{1}+\alpha_{2}+\alpha_{3}>0$ and therefore $\alpha_{1}>-\alpha_{2}-\alpha_{3}>0$, then $\alpha=\left(V_{1}-V_{4}\right) \alpha_{1}+\left(V_{2}-V_{4}\right) \alpha_{2}+\left(V_{3}-V_{4}\right) \alpha_{3}<0$ and (2.1) has either two solutions or no solution.

Case 2: $(-,+,-,-)$. This case like many others can be ruled out by using the geometric interpretation of the $\alpha_{i}$ 's provided by the lemmas. In this case consider the points and lines below.

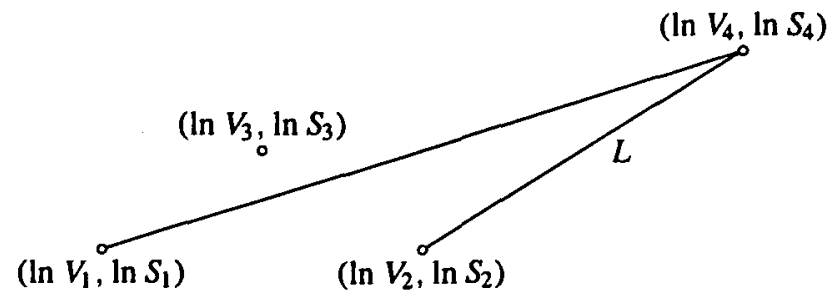


The first minus sign means that $\left(\ln V_{3}, \ln S_{3}\right)$ must be below $L$, a contradiction.

Case 3: $(-,-,+,-)$. This case is ruled out in a completely similar manner to Case 2.

Case 4: $(+,+,-,-)$. This case is ruled out in a completely similar manner to Case 1.

Case 5: $(+,-,+,-)$. This case is ruled out either by the reasoning of Case 1 or by the reasoning of Case 2 .

Case 6: $(-,+,+,-)$. In a similar manner to Case 2 , consider the points and lines shown below.

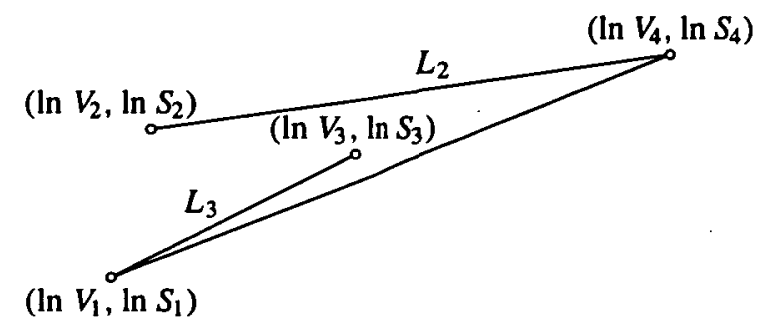

The first minus sign says that $L_{3}$ is below $L_{2}$ but the last minus sign says that $L_{2}$ is below $L_{3}$, a contradiction.

Case 7: $(+,+,+,-)$. Here the same approach as in Case 1 leads to a contradiction.

Case 8. If any one of the first three $\alpha_{i}$ 's is zero, consistent with $\alpha_{1}+\alpha_{2}+\alpha_{3}>0$, the very same arguments as above still apply. If any two of the first three $\alpha_{i}$ 's are zero then all $\alpha_{i}$ 's are zero (using the geometric argument) and therefore $\alpha=0$ and $h=1$ which gives an infinity of solutions.

We now suppose that $\alpha_{1}+\alpha_{2}+\alpha_{3}=0=\alpha_{4}$ and again consider several sub cases.

Case 9: $\alpha_{2}>0$. Using the geometric approach we consider the points and lines shown below.

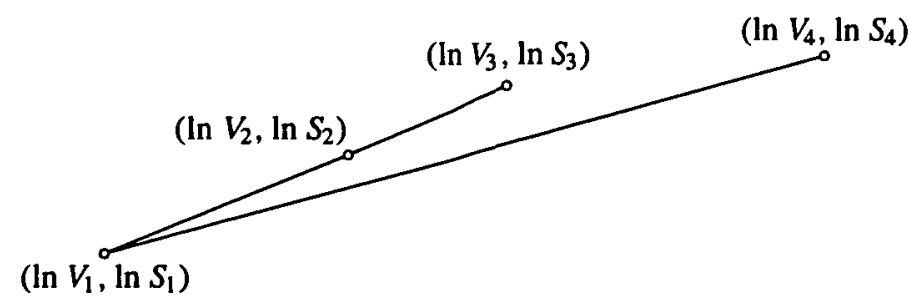

Thus we also have $\alpha_{1}>0$ and hence $\alpha_{3}<0$, a contradiction.

Case 10: $\alpha_{2}<0$. In a completely similar manner we find that $\alpha_{1}<0, \alpha_{3}>0$ also gives a contradiction.

Case 11: $\alpha_{2}=0$. Again by the geometric argument we find that $\alpha_{1}=\alpha_{2}=\alpha_{3}=$ $\alpha_{4}=0$ and $h\left(V_{m}\right)=1$ has an infinity of solutions. 
This disposes of all cases with $\alpha_{1}+\alpha_{2}+\alpha_{3} \geq 0$. If we have $\alpha_{1}+\alpha_{2}+\alpha_{3}<0$ (Figure 2) similar arguments to the above show that there can't be a unique solution here either. The theorem is proved.

\section{Conclusions}

The lack of a unique solution for the parameters $V_{m}, K_{f}, g_{s}$ and $g_{e}$ given any four regular (that is, monotonically arranged) data points $\left(V_{i}, S_{i}\right), i=1,2,3,4$, suggest that Savageau's fractal kinetics model, (1.3), may be deficient as a model of reality.

\section{References}

[1] J. Heidel and J. Maloney, "When can sigmoidal data be fit to a Hill curve?", J. Austral. Math. Soc. Ser. B 41 (1999) 83-92.

[2] J. Heidel and J. Maloney, "An analysis of a fractal Michaelis-Menten curve", J. Austral. Math. Soc. Ser. B 41 (2000) 410-422.

[3] M. A. Lopez-Quintela and J. Casado, "Revision of the methodology in enzyme kinetics: a fractal approach", J. Theor. Biol. 139 (1989) 129-139.

[4] L. Michaelis and M. L. Menten, "Die Kinetik der Invirtinwirkung", Biochem. Z 49 (1913) 333-369.

[5] J. Monod, J. Wyman and J. P. Changeux, "On the nature of allosteric transitions: a plausible model", J. Mol. Biol. 12 (1965) 88-118.

[6] M. A. Savageau, "Michaelis-Menten mechanism reconsidered: implications of fractal kinetics", $J$. Theor. Biol. 176 (1995) 115-124.

[7] E. O. Voit, Canonical nonlinear modeling (Van Nostrand Reinhold, New York, 1991). 